\title{
Bilateral amygdala stimulation reduces avoidance behavior in a predator scent posttraumatic stress disorder model
}

\author{
Bradley A. Dengler, MD, ${ }^{1}$ Shane A. Hawksworth, MD, ${ }^{2}$ Laura Berardo, BA, ${ }^{2}$ lan McDougall, MD, ${ }^{2}$ \\ and Alexander M. Papanastassiou, MD²
} 1Department of Neurosurgery, Walter Reed National Military Medical Center, Bethesda, Maryland; and 2Department of
Neurosurgery, University of Texas Health Science Center at San Antonio, Texas

OBJECTIVE The predator scent model of posttraumatic stress disorder (PTSD) produces prolonged abnormal anxiety and avoidance-like behaviors. Increased basolateral amygdala activity has been shown to correlate with severity of PTSD symptoms in human studies. Modulation of this increased amygdala activity by deep brain stimulation led to improved symptoms in prior studies that used a foot shock model of inducing PTSD. The predator scent model is a different technique that induces long-lasting avoidance behavioral responses by exposing the animal to an inescapable scent of one of its predators. The authors hypothesize that high-frequency stimulation of the bilateral basolateral amygdala will decrease avoidance and anxiety-like behaviors in a predator scent rodent model of PTSD.

METHODS Rodents underwent cat urine exposure in a place preference protocol. Avoidance in the place preference paradigm and anxiety-like behavior in the elevated plus maze were measured before and after high-frequency stimulation.

RESULTS Predator scent exposure resulted in long-term significant avoidance behavior in rodents. Bilateral stimulation significantly decreased avoidance behavior in rodents compared to no stimulation following predator scent exposure. There were no significant differences in anxiety behaviors on the elevated plus maze between stimulated and unstimulated cohorts.

CONCLUSIONS Bilateral stimulation of the basolateral amygdala leads to decreased avoidance behavior compared to controls in a predator scent model of PTSD.

https://thejns.org/doi/abs/10.3171/2018.5.FOCUS18166

KEYWORDS posttraumatic stress disorder; deep brain stimulation; amygdala; anxiety; predator scent; avoidance

$\mathrm{P}$ OSTTRAUMATIC stress disorder (PTSD) is a debilitating psychiatric disorder affecting both civilians and military veterans. It is characterized by symptoms of hyperarousal, impaired cognition, increased anxiety, and avoidance of certain trauma-related cues that began after a life-threatening event. ${ }^{3}$ The disorder is widespread, with a lifetime prevalence of approximately $6.8 \%$ in the United States. ${ }^{11}$ Additionally, $23 \%$ of the 1.64 million veterans returning from conflicts in Iraq and Afghanistan will suffer from PTSD. ${ }^{8}$ Since many returning soldiers will not develop PTSD, it is important to develop a model in which only some animals show avoidance when exposed to a life-threatening stressor. The predator scent model developed by Adamec and Shallow produces prolonged anxiety and avoidance behaviors in rodents exposed to the scent of a male cat's urine. ${ }^{2}$ This model of PTSD is unique in that it leads to robust, long-lasting behavioral changes in rodents, which is in stark contrast to the transient effects of aversive conditioning methods.

The basolateral amygdala processes the sensory and contextual inputs of fear and is involved in fear memory. ${ }^{15}$ Abnormal amygdala activity is found in patients with PTSD. In one study of Vietnam veterans, the bilateral amygdalae were found to be hyperactive in patients

ABBREVIATIONS DBS = deep brain stimulation; PTSD = posttraumatic stress disorder. ACCOMPANYING EDITORIAL DOI: 10.3171/2018.5.FOCUS18266.

SUBMITTED March 31, 2018. ACCEPTED May 14, 2018.

INCLUDE WHEN CITING DOI: 10.3171/2018.5.FOCUS18166. 
TABLE 1. Place preference protocol

\begin{tabular}{cc}
\hline Day & Procedure \\
\hline 1 & $\begin{array}{c}5 \text { mins of free exploration btwn both sides of the place prefer- } \\
\text { ence apparatus }\end{array}$ \\
\hline 2 & 15 mins exposed to one section of the apparatus w/o odor \\
\hline 3 & $\begin{array}{c}15 \text { mins in the opposite section of the apparatus w/ a urine- } \\
\text { soaked sponge }\end{array}$ \\
\hline 4 & 5 mins of free exploration \\
\hline
\end{tabular}

with a diagnosis of PTSD. The severity of the patients' symptoms was correlated with their increased amygdala activity. ${ }^{7}$ Conversely, injury to the amygdala is protective against PTSD. ${ }^{12}$ Despite the evidence of both amygdalae being involved in the pathology of PTSD, the seminal animal study of high-frequency stimulation of the basolateral amygdala to reduce PTSD-like behavior was performed with unilateral stimulation..$^{14}$ These authors demonstrated a reduction in hypervigilant behavior, a treatment effect that was superior to paroxetine in a follow-up study. ${ }^{16}$ The first bilateral amygdala high-frequency stimulation for PTSD in humans was reported in 2015. ${ }^{13}$

We hypothesized that high-frequency stimulation of the bilateral basolateral amygdala would result in decreased avoidance and anxiety-like behaviors in the predator scent, rodent PTSD model. In contrast to prior studies, we hope to demonstrate the efficacy of bilateral high-frequency stimulation in a long-term animal model of PTSD, the predator scent model.

\section{Methods}

\section{Experimental Design}

After 4 days of acclimation the animals underwent a place preference protocol, as described by Edwards et al. ${ }^{5}$ (Table 1). Baseline avoidance behavior was determined after exposure to a predator scent. Avoidance of traumarelated settings along with increased anxiety are two of the main features required for the clinical diagnosis of PTSD. The avoidance paradigm in which a rat is exposed to a stress-inducing scenario such as a predator scent in a specific environment mimics the same type of cues that humans would encounter leading to their PTSD. Additionally, the elevated plus maze is a well-documented measure of anxiety in a rodent; the more anxious animals will avoid the open arms because they feel more vulnerable to threats. A cutoff criterion was used to select animals to continue to the rest of the protocol. These animals were randomly assigned to receive bilateral, unilateral, or no stimulation. A fourth group of control animals underwent the same place preference protocol but was never exposed to the predator scent. Four days after undergoing avoidance testing, all animals were tested on the elevated plus maze. The following day, all animals were implanted with bilateral amygdala stimulation electrodes and given 1 week to recover. Then they underwent high-frequency stimulation for 4 hours a day for 7 days before undergoing repeat avoidance testing and elevated plus maze testing. The repeat testing occurred the day after their last stimulation period. The day after the repeat behavior testing, all animals were euthanized (Fig. 1).

\section{Animals}

Seventy-eight 10- to 12-week-old Lewis rats weighing approximately $350-400 \mathrm{~g}$ were used for this work. The animals were housed in groups of 3 rats in one cage and had a standard 12-hour light/dark cycle with lights on at 07:30. The animals were acclimated for 4 days before any experimental manipulations were undertaken, and had free access to food and water throughout the experiment. This experimental protocol was reviewed and approved by the University of Texas Health Science Center at San Antonio Institutional Animal Care and Use Committee.

\section{Place Preference}

Animals underwent a 4-day place preference protocol. The apparatus consisted of 2 large boxes $(50 \mathrm{~cm} \times 50 \mathrm{~cm} \times$ $40 \mathrm{~cm}$ ) connected by a $10-\mathrm{cm}$-wide pathway (Fig. 2). One box was made of black Plexiglas with a wire mesh floor and the other was made of white Plexiglas with a smooth floor. On day 1, the animals were started in the white box and underwent 5 minutes of free exploration. On day 2, they were confined for 15 minutes to the box they did not

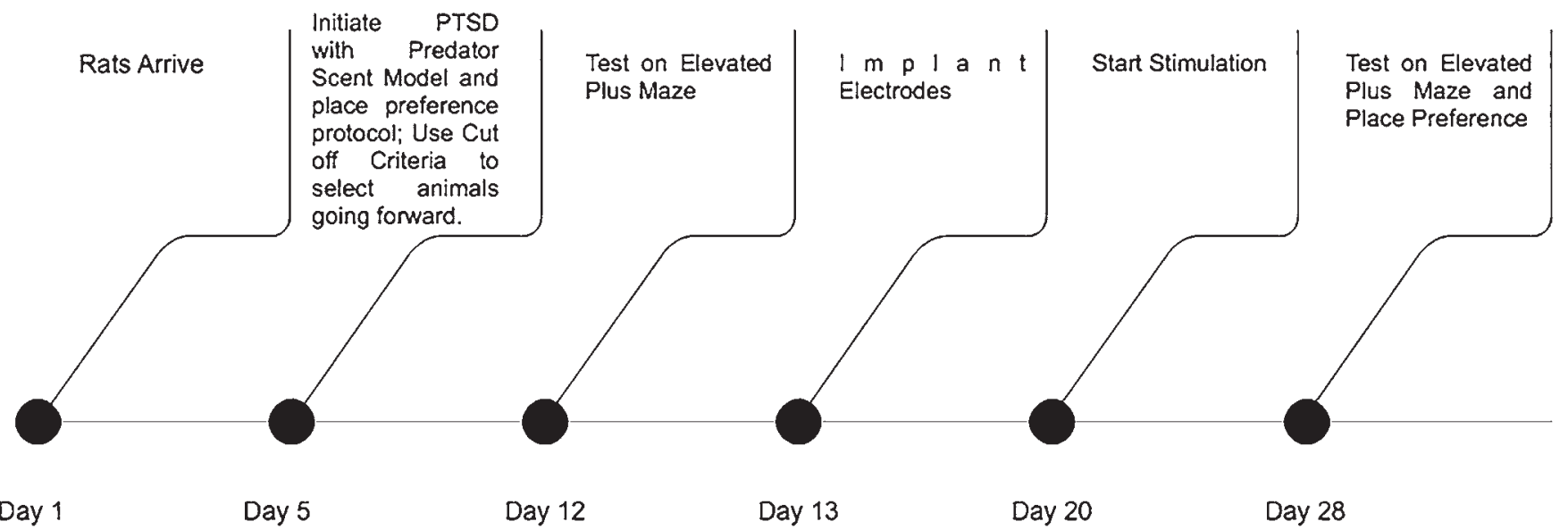

FIG. 1. Timeline of the experimental protocols, starting from when rats arrive. 


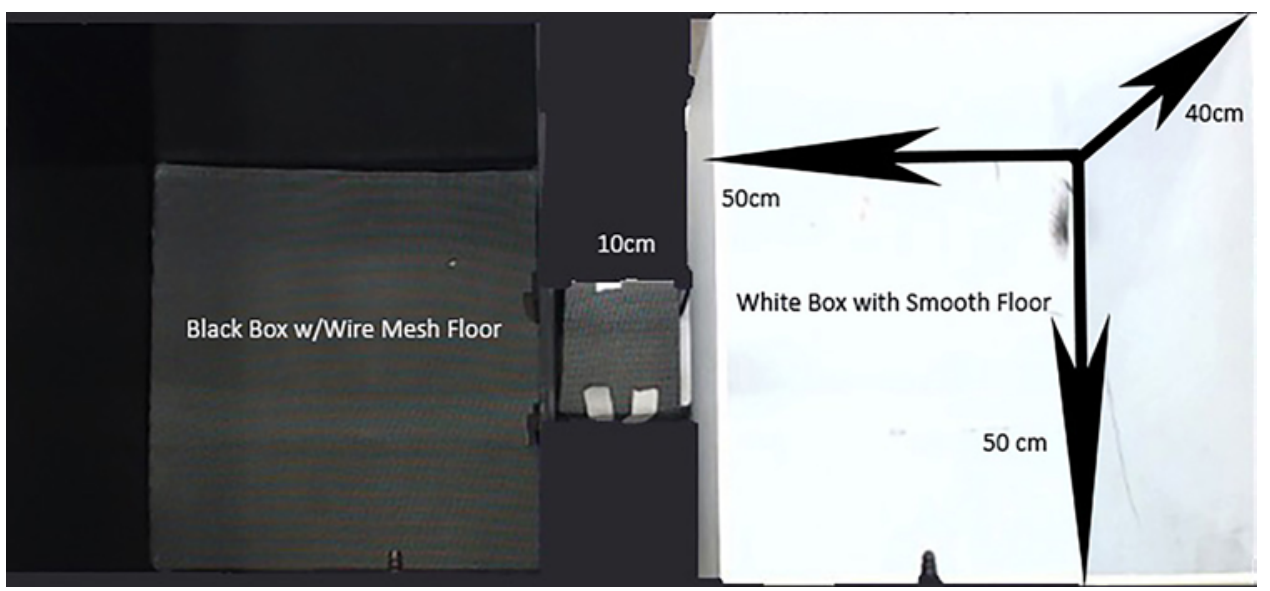

FIG. 2. Photograph of the place preference apparatus, with measurements.

favor on day 1 . On day 3 , they were confined to the opposite chamber and underwent 15 minutes of predator scent exposure. On day 4, each animal was started out in the white chamber and was allowed 5 minutes of free exploration (Table 1). Time spent in each box was counted when all 4 paws of the animals made it into the box.

\section{Predator Scent Exposure}

Rats were exposed to a sponge soaked with $10 \mathrm{ml}$ of fresh male cat urine (Liberty Research). They were exposed for a total of 15 minutes and were able to freely move around.

\section{Elevated Plus Maze}

The elevated plus maze was first tested 5 days after the rats' predator scent exposure, and then again the day after their last day of stimulation. The maze consisted of $4 \mathrm{arms}$ situated $90^{\circ}$ apart, each $50 \mathrm{~cm}$ in length and $10 \mathrm{~cm}$ wide. Two of the arms are enclosed by walls $40 \mathrm{~cm}$ high. The maze is elevated off the ground by $50 \mathrm{~cm}$ (Fig. 3). The animals were placed into the middle of the maze facing an open arm and were given 5 minutes of free exploration time, and they were recorded and scored by Any-Maze video tracking software. Open arm and closed arm entries and time were recorded. Anxiety index was calculated as $1-\{[$ (time in open arm/time in maze) + (entry to open arm/total exploration in maze)]/2\}. ${ }^{2}$

\section{Surgical Procedure}

General anesthesia was induced and continued with isoflurane. The animal was placed into a stereotactic frame. The hair overlying the scalp was clipped and the area was prepared with iodine. A midline incision was made over the scalp to expose the skull. A high-speed drill was used to make a burr hole overlying the basolateral amygdala at $-2.4 \mathrm{~mm}$ from bregma and $4.8 \mathrm{~mm}$ lateral to the sagittal suture bilaterally. An 8.5-mm monopolar stimulating electrode (Plastics One) was then lowered through the burr hole until the plastic cap was flush with the skull. Two jeweler's screws were then placed into the skull around each electrode and the whole skull was covered with dental cement.

\section{Stimulation}

Animals were moved into individual cages each day for their stimulation. The stimulation cables were attached to each animal with enough length for them to be able to freely move around the cage. Each underwent stimulation for 4 hours a day for 7 days. The stimulation settings were monopolar, $120-\mu$ sec pulse width, $160-\mathrm{Hz}$ frequency, and $30 \mathrm{~mA}$. High-frequency stimulation was completed using the model STG4008 1.6-mA, 8-channel, general-purpose stimulus generator, and programming was completed using the MC_Stimulus II software (Multi Channel Systems MCS GmbH, 2016). Unilaterally stimulated animals received right-sided stimulation only. All animals were connected to the stimulator for 4 hours each day, receiving electrical current based on their assigned cohort (unilateral right side, bilateral, no stimulation).

\section{Place Avoidance Testing}

The day after the last round of stimulation, each animal was placed back into the previously described avoidance chamber, starting in the white side. They were allowed 5 minutes of free exploration throughout the apparatus. Their movements were recorded on a video camera and total time spent in each side was scored later. Time was counted when all 4 paws of the animal were completely within the box.

\section{Histology}

At the conclusion of the experiments the animals were deeply anesthetized with isoflurane, and then transcardiac perfusion was performed with normal saline followed by $4 \%$ paraformaldehyde. The rats' brains were dissected, cryoprotected with a $30 \%$ sucrose solution, and flash frozen. The brains were then frozen and sliced on a cryostat to $30-\mu \mathrm{m}$ slices. Slices underwent Nissl staining and were viewed under a light microscope to confirm electrode placement.

\section{Statistical Analysis}

Quantitative data are reported as the mean $\pm \mathrm{SD}$. Microsoft Excel for Mac version 15.32 was used to perform 


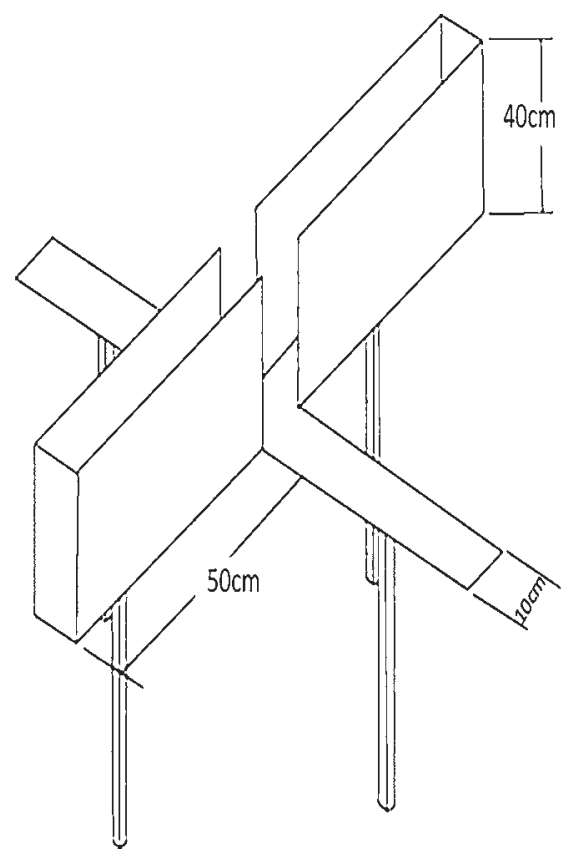

FIG. 3. Diagram of the elevated plus maze device.

a Student t-test (Microsoft Corp., 2017). Remaining statistical analyses were performed using GraphPad Prism version 7.0c for Mac OS X (GraphPad Software, 2017). One-way ANOVA was used to determine statistical significance.

\section{Results \\ Cutoff Criteria}

Animals exposed to the predator scent spent an average of $34.3 \pm 68.9$ seconds less time in the avoidance chamber than did controls. Using 1 SD from the mean, a cutoff criterion was created to identify those animals with a significant response to the predator scent. Of the 68 rodents exposed to the predator scent, 25 demonstrated this significant response. Two additional animals were removed from analysis due to misplaced electrodes on histological investigation, as described below. One of the misplaced electrodes was in a control rat, and the second one was the left-sided electrode in the unilateral right-side stimulation group.

\section{Place Avoidance}

Each of the 3 treatment groups exposed to predator scent spent significantly less time in the avoidance chamber following predator scent exposure when compared to the unexposed control group (Fig. 4). This treatment effect was not significantly different among the 3 groups exposed to the predator scent. The effect of the predator scent on avoidance-like behavior was sustained at poststimulation testing, 21 days after the initial predator scent exposure. During the poststimulation testing, the no stimulation, no predator scent group spent significantly more time in the avoidance chamber than the no stimulation, predator scent group (214 \pm 48 seconds vs $119 \pm 35$ seconds, $\mathrm{p}=0.0028)$.

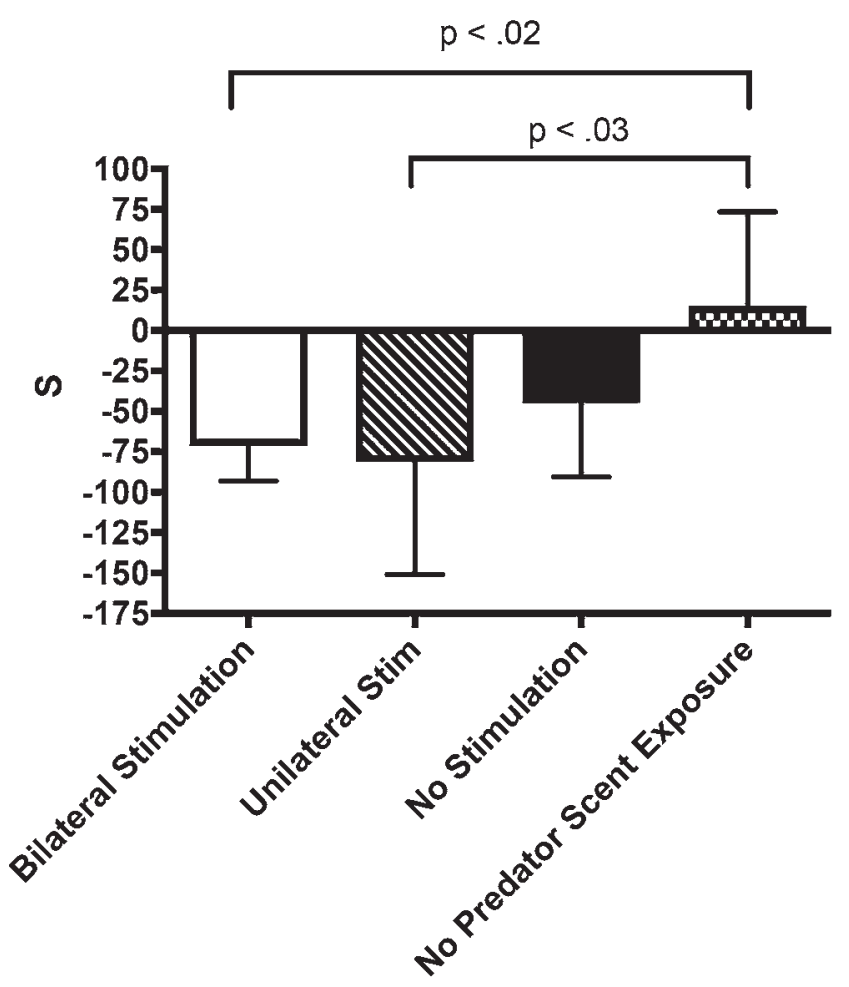

FIG. 4. This graph represents the difference in time spent in the avoidance chamber before and after the predator scent exposure. The delta value is calculated by taking the time spent in the avoidance chamber after the predator scent exposure and subtracting the time spent before the exposure. ANOVA: $p<0.04 . s=$ seconds.

Following stimulation, the bilateral stimulation group spent significantly more time in the avoidance chamber than the no stimulation, predator scent group or the no stimulation, no predator scent exposure group (Fig. 5). Response to unilateral stimulation and no stimulation did not significantly differ from control. Bilateral and unilateral stimulation both increased time in the avoidance chamber, but did not vary significantly from each other.

\section{Elevated Plus Maze}

Animals underwent two elevated plus maze trials. Changes in time spent in the open arm, time spent in the closed arm, and the anxiety index were determined. There were no significant differences among treated groups (Table 2). Four animals could not complete an elevated plus maze trial because they left the platform and thus were excluded from this analysis. There were no significant differences of mean change in the time spent in open or closed arms or change in the anxiety index in treatment groups before and after stimulation by 1-way ANOVA.

\section{Histological Analysis}

Nissl staining was completed in 16 rodents; 22 electrode paths were visualized (Fig. 6). It was determined that 2 electrodes were misplaced, suggesting an overall accuracy of electrode placement into the basolateral amygdala of $91 \%$. 


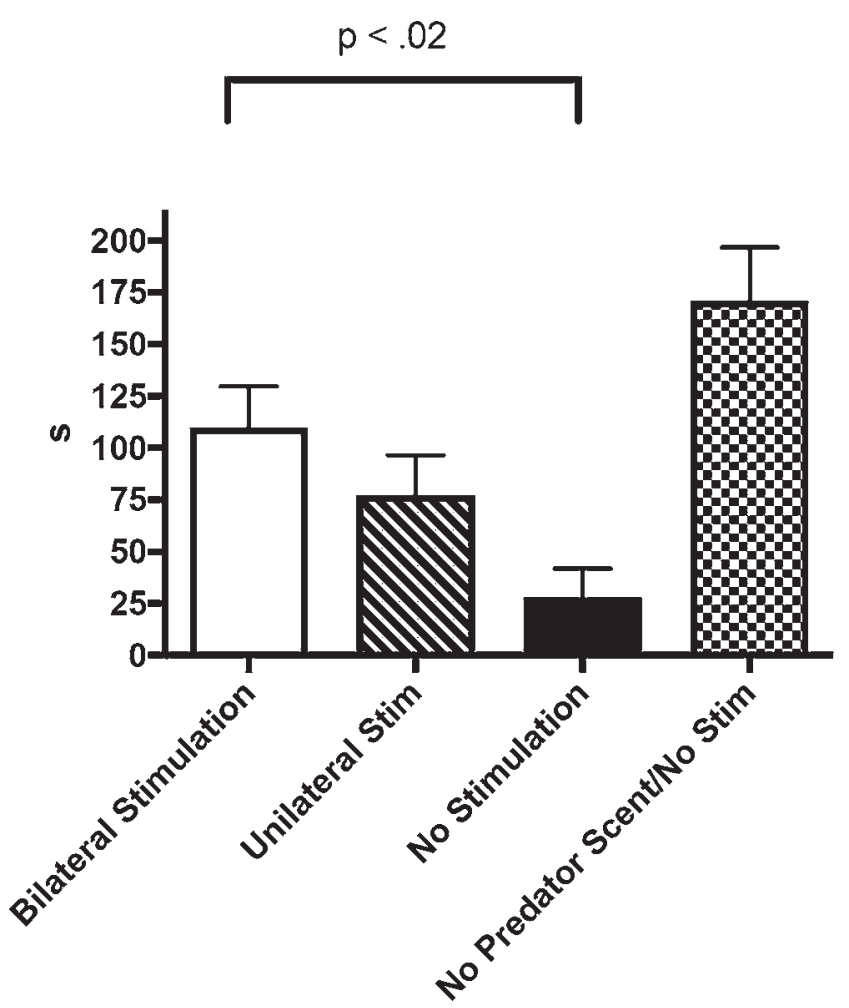

FIG. 5. This graph represents the total time spent in the previously avoided chamber after 1 week of high-frequency stimulation (stim) ANOVA: $p<0.05$.

\section{Discussion}

The most current literature suggests that PTSD is an abnormal response to conditioned fear. ${ }^{17}$ In this classic pavlovian framework, a conditioned stimulus (a bell) is paired with an unconditioned stimulus (meat powder), leading to an unconditioned response (salivating). Eventually, the conditioned stimulus produces the conditioned response without the presence of the unconditioned stimulus. With continued use of the conditioned stimulus without its pairing with the unconditioned stimulus, the animal will eventually unlearn the pairing and stop salivating in the classic pavlovian description. In pathological PTSD, the environment and contextual cues (conditioned stimulus) are paired with the traumatic event (unconditioned stimulus), leading to long-term anxiety symptoms and avoidance behaviors. Patients with PTSD will continue to have these symptoms even after they have experienced the

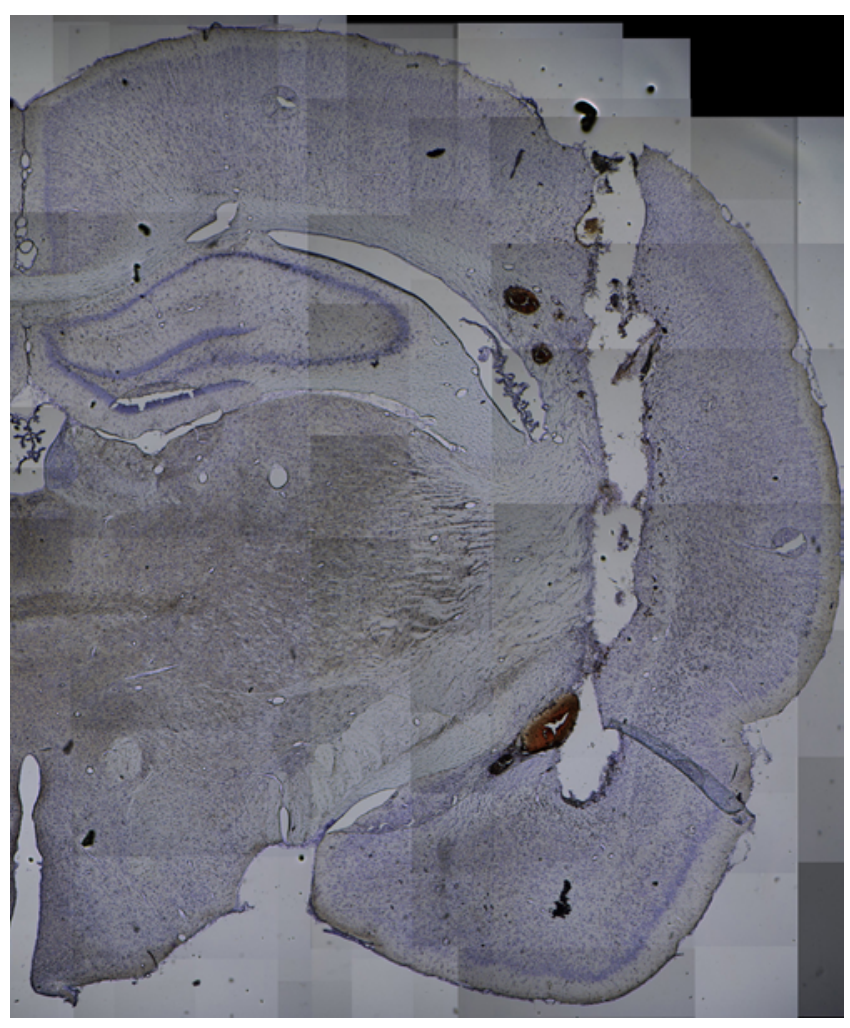

FIG. 6. Coronal section of rat brain showing location of implanted electrode. Nissl stain, original magnification $\times 20$.

conditioned stimulus multiple times without the presence of a life-threatening event. The amygdala is central in the process of linking emotional memories to their behavioral responses..$^{16}$ Multiple studies have linked amygdala damage to protection against developing PTSD..$^{12}$ Additionally, patients with PTSD showed increased amygdala activity in functional MRI studies. ${ }^{7}$ Because the amygdala appears to be central in the process of linking emotional responses and memories and has increased activity in PTSD, modulating it by way of deep brain stimulation (DBS) seems to be a viable therapy.

This is the first study to look at high-frequency stimulation of the bilateral rodent basolateral amygdala to reduce avoidance behavior after predator scent exposure. Rodents who underwent bilateral high-frequency stimulation of the basolateral amygdala demonstrated a significant decrease in avoidance behavior. The predator scent model is a wellestablished paradigm for inducing behavioral respons-

TABLE 2. No change in anxiety-like behavior on the elevated plus maze after stimulation

\begin{tabular}{lcccrc}
\hline \multicolumn{1}{c}{ Parameter } & Unilat Stimulation & Bilat Stimulation & No Stimulation & No Predator Scent & $p$ Value \\
\hline No. of rats & 3 & 5 & 4 & \multicolumn{1}{c}{7} & \\
\hline$\Delta$ open time $(\mathrm{sec})$ & $27.2 \pm 55.5$ & $-13.6 \pm 34.9$ & $9.6 \pm 11.5$ & $-19.6 \pm 58.1$ & 0.4064 \\
\hline$\Delta$ closed time $(\mathrm{sec})$ & $-3.9 \pm 118.2$ & $16.4 \pm 41.8$ & $-5.4 \pm 34.8$ & $31.5 \pm 62.1$ & 0.8238 \\
\hline$\Delta$ anxiety index & $-0.4 \pm 0.163$ & $0.018 \pm 0.087$ & $-0.026 \pm 0.031$ & $0.027 \pm 0.112$ & 0.7444 \\
\hline
\end{tabular}

Unless otherwise indicated, values are expressed as the mean \pm SD.

${ }^{*} \mathrm{~A}$ total of 78 rats were used to acquire the numbers in each group; the rest did not meet the cutoff criteria and did not continue the protocol. 
es similar to the ones seen in human PTSD, especially anxiety and avoidance behaviors., ${ }^{4,9}$ Thus far, the Gilpin laboratory has been the only other one to expand on the predator scent by using it in a place preference paradigm to explore avoidance behavior. . $^{5,10,18,19}$

In contrast to prior studies using the foot shock model, the predator scent model used in this study is a speciesrelevant, life-threatening, inescapable stress that leads to lasting effects on avoidance behavior. In our study the avoidance behavior of the controls lasted well into the 3rd week, which confirms the reports by other authors showing long-lasting effects. ${ }^{1,2}$ Given the short lifespan of rodents, 3 rodent weeks may be equivalent to 18 human months. ${ }^{2}$ The chronic avoidance behavior was improved with only 4 hours a day of stimulation for 1 week, providing more evidence that modulation of the basolateral amygdala may be beneficial in PTSD. Additionally, our model more closely resembles actual clinical practice because the animals were implanted only when they showed avoidance behavior, and there was a significant gap between implantation and retesting, ensuring that this was a chronic condition.

In contrast to the improved avoidance behavior, highfrequency stimulation of the basolateral amygdala did not reduce anxiety-like behavior as measured on the elevated plus maze. This is in agreement with a prior study of high-frequency unilateral amygdala stimulation, which similarly demonstrated no difference in anxiety-like behavior..$^{16}$ In this study, paroxetine administration did lower anxiety-like behavior compared to controls. These findings may support the need for combined therapy-highfrequency stimulation plus medications or other modalities-to adequately treat all abnormal PTSD behaviors, and the hypothesis that basolateral amygdala stimulation may be a treatment for patients with predominant avoidance behaviors. Further study is required to help elucidate a neural target for modulation that will adequately treat the anxiety component of PTSD. In our study, Table 2 shows that there was a possible trend toward increased anxiety in the bilateral stimulation group, with more time spent in the closed arms. The study was underpowered to look at this effect, so more studies should be done to ensure that this is not a real effect before more human trials are started. There might be a small effect toward increasing anxiety, but more animals would be needed to show this, given the high variance of the data.

In our study, the unilateral stimulation group did not reach statistical significance when compared to the nostimulation group, but there was no statistical difference between the unilateral and bilateral stimulation groups. This means that there is probably some effect to unilateral stimulation that is further multiplied by bilateral stimulation. The 2 rodents with misplaced electrodes showed some increased time in the avoidance chamber, suggesting that there might be a small effect from an amygdala lesion. Because the unilateral stimulation group did not rise to statistical significance, but Langevin and colleagues had shown results with only unilateral stimulation, this suggests that not all PTSD animal models are the same.13,14 Additional work should also be performed to evaluate how unimplanted rats would perform after the predator scent exposure to help define the possible lesioning effect. Pre- sumably these rats would show more avoidance than the predator scent-exposed group who did not undergo implantation.

In our study, we did not group rats based on the severity of their response to the predator scent model, but there were certainly rats who responded more severely than others, suggesting that certain genetic makeups could influence development of PTSD or symptom severity. Using the predator scent model provides an opportunity to study this variable response and to look at such things as genetic makeup or epigenetic markers. These data can then be used to plan future human trials by helping to better select patients for implantation of DBS electrodes, thereby increasing the likelihood of a successful trial. For instance, if it can be shown that certain epigenetic markers respond better to stimulation, then these same markers can be measured in humans, and those patients targeted for implantation.

\section{Conclusions}

These results suggest that avoidance behavior in a predator scent model of PTSD can be improved with highfrequency stimulation of the basolateral amygdala. This study provides more promising data that DBS might be an effective treatment for complex, debilitating, refractory PTSD.

\section{Highlights}

The following are the highlights of this study. 1) A predator scent model was used to create significant avoidance behavior. 2) Bilateral basolateral amygdala stimulation reduced avoidance behavior. 3) Anxiety-like behaviors were not significantly altered by stimulation of the basolateral amygdala.

\section{Acknowledgments}

Thanks to Naomi Sayre, $\mathrm{PhD}$, and Shane Sprague for their assistance and feedback on this project.

\section{References}

1. Adamec R, Hebert M, Blundell J, Mervis RF: Dendritic morphology of amygdala and hippocampal neurons in more and less predator stress responsive rats and more and less spontaneously anxious handled controls. Behav Brain Res 226:133-146, 2012

2. Adamec RE, Shallow T: Lasting effects on rodent anxiety of a single exposure to a cat. Physiol Behav 54:101-109, 1993

3. American Psychiatric Association: Diagnostic and Statistical Manual of Mental Disorders, ed 5. Washington, DC: American Psychiatric Association, 2013

4. Cohen H, Zohar J: An animal model of posttraumatic stress disorder: the use of cut-off behavioral criteria. Ann N Y Acad Sci 1032:167-178, 2004

5. Edwards S, Baynes BB, Carmichael CY, Zamora-Martinez ER, Barrus M, Koob GF, et al: Traumatic stress reactivity promotes excessive alcohol drinking and alters the balance of prefrontal cortex-amygdala activity. Transl Psychiatry 3:e296, 2013

6. Elder GA, Dorr NP, De Gasperi R, Gama Sosa MA, Shaughness MC, Maudlin-Jeronimo E, et al: Blast exposure induces post-traumatic stress disorder-related traits in a rat model of 
mild traumatic brain injury. J Neurotrauma 29:2564-2575, 2012

7. Etkin A, Wager TD: Functional neuroimaging of anxiety: a meta-analysis of emotional processing in PTSD, social anxiety disorder, and specific phobia. Am J Psychiatry 164:1476-1488, 2007

8. Fulton JJ, Calhoun PS, Wagner HR, Schry AR, Hair LP, Feeling N, et al: The prevalence of posttraumatic stress disorder in Operation Enduring Freedom/Operation Iraqi Freedom (OEF/OIF) veterans: a meta-analysis. J Anxiety Disord 31:98-107, 2015

9. Goswami S, Samuel S, Sierra OR, Cascardi M, Paré D: A rat model of post-traumatic stress disorder reproduces the hippocampal deficits seen in the human syndrome. Front Behav Neurosci 6:26, 2012

10. Itoga CA, Roltsch Hellard EA, Whitaker AM, Lu YL, Schreiber AL, Baynes BB, et al: Traumatic stress promotes hyperalgesia via corticotropin-releasing factor-1 receptor (CRFR1) signaling in central amygdala. Neuropsychopharmacology 41:2463-2472, 2016

11. Kessler RC, Berglund P, Demler O, Jin R, Merikangas KR, Walters EE: Lifetime prevalence and age-of-onset distributions of DSM-IV disorders in the National Comorbidity Survey Replication. Arch Gen Psychiatry 62:593-602, 2005 (Erratum in Arch Gen Psychiatry 62:768, 2005)

12. Koenigs M, Huey ED, Raymont V, Cheon B, Solomon J, Wassermann EM, et al: Focal brain damage protects against posttraumatic stress disorder in combat veterans. Nat Neurosci 11:232-237, 2008

13. Langevin JP, Chen JW, Koek RJ, Sultzer DL, Mandelkern MA, Schwartz HN, et al: Deep brain stimulation of the basolateral amygdala: targeting technique and electrodiagnostic findings. Brain Sci 6:6, 2016

14. Langevin JP, De Salles AA, Kosoyan HP, Krahl SE: Deep brain stimulation of the amygdala alleviates post-traumatic stress disorder symptoms in a rat model. J Psychiatr Res 44:1241-1245, 2010

15. LeDoux JE: Emotion circuits in the brain. Annu Rev Neurosci 23:155-184, 2000
16. Stidd DA, Vogelsang K, Krahl SE, Langevin JP, Fellous JM: Amygdala deep brain stimulation is superior to paroxetine treatment in a rat model of posttraumatic stress disorder. Brain Stimul 6:837-844, 2013

17. VanElzakker MB, Dahlgren MK, Davis FC, Dubois S, Shin LM: From Pavlov to PTSD: the extinction of conditioned fear in rodents, humans, and anxiety disorders. Neurobiol Learn Mem 113:3-18, 2014

18. Whitaker AM, Farooq MA, Edwards S, Gilpin NW: Posttraumatic stress avoidance is attenuated by corticosterone and associated with brain levels of steroid receptor co-activator-1 in rats. Stress 19:69-77, 2016

19. Whitaker AM, Gilpin NW: Blunted hypothalamo-pituitary adrenal axis response to predator odor predicts high stress reactivity. Physiol Behav 147:16-22, 2015

\section{Disclosures}

The authors report no conflict of interest concerning the materials or methods used in this study or the findings specified in this paper. The views expressed in this article are those of the author and do not reflect the official policy of the Department of the Army, Department of Defense, or US government.

\section{Author Contributions}

Conception and design: Dengler, Papanastassiou. Acquisition of data: Dengler, Hawksworth, Berardo, McDougall. Analysis and interpretation of data: Dengler, Hawksworth. Drafting the article: Dengler, Hawksworth. Critically revising the article: Dengler, Hawksworth. Reviewed submitted version of manuscript: Dengler. Approved the final version of the manuscript on behalf of all authors: Dengler. Statistical analysis: Dengler. Study supervision: Papanastassiou.

\section{Correspondence}

Bradley A. Dengler: Walter Reed National Military Medical Center, Bethesda, MD. bdengler86@gmail.com. 